\title{
Correlation Between Radiosensitizing Activity and the Stereo-structure of the TX-2036 Series of Molecules
}

\author{
KAZUTO OHKURA ${ }^{1}$, ATSUSHI TABATA ${ }^{2}$, YOSHIHIRO UTO ${ }^{2}$ and HITOSHI HORI ${ }^{2}$ \\ ${ }^{1}$ Graduate School of Pharmaceutical Sciences, Suzuka University of Medical Science, Suzuka, Japan; \\ ${ }^{2}$ Department of Biological Science and Technology, Life System, Institute of Technology and Science, \\ The University of Tokushima Graduate School, Tokushima, Japan
}

\begin{abstract}
Background/Aim: The stereo-configuration $(R-, S$-configuration) of chiral-2-nitroimidazole derivatives alters their radiosensitizing activity. This study aimed at examining the molecular features of these enantiomers by molecular simulation techniques. Materials and Methods: A series of 2-nitroimidazole-based radiosensitizer TX-2036 molecules were synthesized, and their profiles were examined using molecular structural analysis such as conformation analysis, molecular orbital analysis, and electrostatic potential analysis. Results: R-configured TXs (TX-2043, -2030, -2036) had a weaker radiosensitizing activity than S-configured TXs (TX-2044, -2031, -2037), and R-compounds had a small minus electrostatic potential (ESP) field in the cyclopentene-1,3-dione region. S-configured TX-2046 had weaker radiosensitizing activity than R-configured TX-2045, and TX-2046 had a small minus ESP field as well as R-configured TX-2043, -2030, 2036. Conclusion: The cyclopentene-1,3-dione involved in the small minus ESP field affected the radiosensitizing activity of the TX-2036 series of molecules.
\end{abstract}

We recently reported the design, synthesis, and evaluation of racemic and enantiomerically pure chiral haloacetylcarbamoyl2-nitroimidazoles, including chloro- and bromo-derivatives, as antiangiogenic hypoxic cell radiosensitizers (1). In the tumor microenvironment, there are soft nucleophiles such as nonprotein thiols and thiol proteases. Therefore, we developed a strategy to design 2-nitroimidazole derivatives that incorporate a soft electrophile, the aminomethylenecyclopentenedione moiety, as a new anti-angiogenic and anti-tumor functional group. We considered two potential benefits of a chiral center

Correspondence to: Professor Kazuto Ohkura, Graduate School of Pharmaceutical Sciences, Suzuka University of Medical Science, 3500-3 Minamitamagaki-cho, Suzuka, Mie 513-8670, Japan. Tel: +81 593400611, Fax: +81 593681271, e-mail: kohkura@suzuka-u.ac.jp

Key Words: Molecular design, structure, radiosensitizing activity, TX-2036. for our hypoxic cell radiosensitizers: First, this would provide us with two molecular structures that have different biological activities from the same synthetic route and, second, each enantiomer would possess specific pharmacokinetic and pharmacodynamic properties. Herein, we present our design, syntheses, and biological evaluation of new 2-nitroimidazolebased anti-angiogenic hypoxic cell radiosensitizers that incorporate the 2-aminomethylene-4-cyclopentene-1,3-dione moiety as an anti-angiogenic pharmacophoric descriptor (2).

\section{Materials and Methods}

Energy analysis of the TX-2036 series. TX-2036 and TX-2036 derivatives were designed and synthesized as previously described (2). Conformation analysis of the synthesized TX-2036 series of molecules was performed using CAChe-Conflex (Fujitsu Inc., Tokyo, Japan), and energy profiles were obtained as previously described $(3,4)$. Solvation free energy (stereo-hydrophobicity: $\mathrm{dGW}$ ) was determined using Mopac (Fujitsu Inc.) as previously described $(5,6)$. Electrostatic potential (ESP) fields of molecules from the TX-2036 series were analyzed using CAChe (Fujitsu Inc.) as previously described (7). ESP field analysis was performed for a minimum energy conformer of each TX-2036 derivative.

In vitro radiosensitizing assay. In vitro radiosensitization was measured in EMT6/KU single cells under hypoxic conditions as previously described (8). Enhancement ratios (ERs) were determined from the ratio of radiation doses required to reduce the surviving fraction of EMT6/KU cells to 1\%. Usually, each ER value of radiosensitizer was obtained from survival curves consisting of four or five points per curve and converted based on the ER value of etanidazole $(\mathrm{ER}=1.72)(2)$.

\section{Results}

Molecular profiles of TX-2036 derivatives. The structures of TX-2036 derivatives are shown in Figure 1. These compounds had a chiral center and were classified into $R$-configurations (TX-2043, -2030, -2036) or $S$-configuration (TX-2044, -2031, -2037). The radiosensitizing activity of $R$-configured TX-2043 (ER=1.80) was weaker than that of $S$-configured TX-2044 (ER=2.00) (Table I). In other 
<smiles>[R]OC[C@H](Cn1ccnc1[N+](=O)[O-])OC(=O)c1ccc(NC=C2C(=O)C=CC2=O)cc1</smiles>

1. TX-2043: R-configuration, $\mathrm{R}=$ methyl

2. TX-2044: $\mathrm{S}$-configuration, $\mathrm{R}=$ methyl

3. TX-2030: R-configuration, $\mathrm{R}=$ tert-butyl

4. TX-2031: $\mathrm{S}$-configuration, $\mathrm{R}=$ tert-butyl

5. TX-2036: R-configuration, $\mathrm{R}=p$-tert-butyl

6. TX-2037: S-configuration, $\mathrm{R}=p$-tert-butyl

\section{1. $\mathrm{TX}-2045$ : $\mathrm{R}$-configuration, $\mathrm{R}=$ methyl \\ 12. TX-2046: S-configuration, $\mathrm{R}=$ methyl}

Figure 1. Structure of TX-2036 derivatives.

TX-pairs, the radiosensitizing activity of $R$-configured TXs was weaker than that of $S$-configured TXs (TX-2030 (1.68) vs. TX-2031 (1.80), TX-2036 (1.79) vs. TX-2037 (1.93)). Moreover, the radiosensitizing activity of 2,2,2,-trifluoroacetylamino group involved in the $R$-configured TX-2045 (2.02) was stronger than that of $S$-configured TX-2046 (1.84).

The energy profiles of enantiomer pairs (e.g. TX-2030, -2031 pair (13.8 - $27.0 \mathrm{kcal} / \mathrm{mol})$, TX-2036, -2037 pair (4.7-18.6 $\mathrm{kcal} / \mathrm{mol}), \mathrm{TX}-2043,-2044$ pair (8.8-22.9 kcal/mol), TX-2045, -2046 pair $(60.7-67.3 \mathrm{kcal} / \mathrm{mol}))$ were almost unchanged by their stereo-configuration during conformation analysis (open circles in Figure 2). Solvation free energy (an index of stereohydrophobicity) profiles of these enantiomer pairs did not differ (e.g. TX-2030, -2031 pair (-218.9 - -194.4 kJ/mol), TX2036, -2037 pair (-229.1 - -195.1 kJ/mol), TX-2043, -2044 pair (-232.6 - -204.5 kJ/mol), TX-2045, -2046 pair (-208.7 - -178.5 $\mathrm{kJ} / \mathrm{mol})$ ) (closed circles in Figure 2).

Electrostatic potential fields of TX-2036 derivatives. For the TX-2030, -2031 enantiomer pair and the TX-2036, -2037 pair, the electrostatic potential (ESP) fields were similar, but small minus ESP fields were observed in the cyclopentene1,3-dione portion of $R$-configured TX-2030 and TX-2036 (arrows in Figure 3). For the TX-2043, -2044 pair, the ESP fields were similar, but two small minus ESP fields were observed in the cyclopentene-1,3-dione portion of $R$ configured TX-2043 (arrows). For the TX-2045 and -2046 pair, two small minus fields were observed in the 2,2,2trifluoroacetylamino group (arrows) of $S$-configured TX-
Table I. Radiosensitizing activity of 2-nitroimidazole-aminomethylenecyclopentenediones.

\begin{tabular}{lc}
\hline Compounds & ER (concentration) \\
\hline Etanidazole & $1.72(1 \mathrm{mM})$ \\
1 (TX-2043) & $1.80(1 \mu \mathrm{M})$ \\
2 (TX-2044) & $2.00(1 \mu \mathrm{M})$ \\
$3(\mathrm{TX}-2030)$ & $1.68(1 \mu \mathrm{M})$ \\
4 (TX-2031) & $1.80(1 \mu \mathrm{M})$ \\
$5(\mathrm{TX}-2036)$ & $1.79(1 \mu \mathrm{M})$ \\
6 TX-2037) & $1.93(1 \mu \mathrm{M})$ \\
11 TX-2045) & $2.02(1 \mu \mathrm{M})$ \\
$12(\mathrm{TX}-2046)$ & $1.84(1 \mu \mathrm{M})$ \\
\hline
\end{tabular}

ER (concentration): Enhancement ratio at corresponding concentration.

2046. TX-2036 derivatives with these small minus fields indicated weaker radiosensitizing activity than in the corresponding stereoisomers (Table I).

\section{Discussion}

Synthesized TX-2036 S-derivatives (TX-2044, -2031, -2037) had stronger radiosensitizing activity than the corresponding $R$ derivatives (TX-2043, -2030, -2036). Conformation profile and stereohydrophobicity analysis did not reveal a difference between the $S$ - and $R$-configured TX-2036 derivatives. In the electrostatic potential field analysis, small minus ESP fields were 

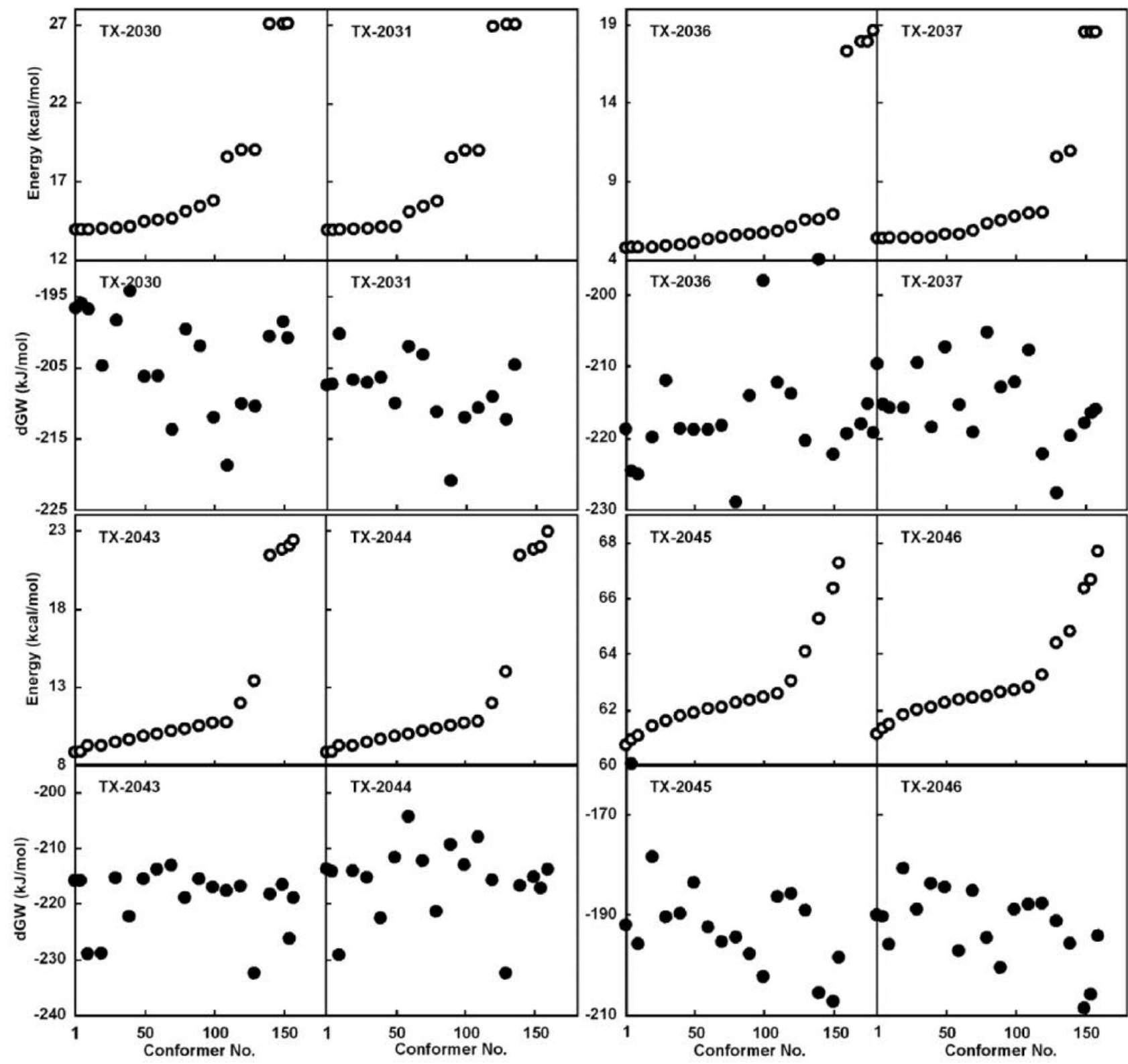

Figure 2. Energy profiles of conformation analysis of TX-2036 derivatives. Heat formation energy profiles of TX-2030, TX-2031, TX-2036, TX-2037, $T X-2043, T X-2044, T X-2045, T X-2046$ are shown in upper panels. Solvation free energy (stereo-hydrophobicity (dGW)) profiles of TX-2030, TX-2031, TX-2036, TX-2037, TX-2043, TX-2044, TX-2045, TX-2046 are shown in lower panels.

observed only in $R$-configurations (TX-2043, -2030, -2036) in the cyclo-pentene-1, 3-dione region. These small minus fields influenced radiosensitizing activity. The radiosensitizing activity of TX-2046 ( $S$-configuration) with a small ESP field was lower than that of TX-2045 ( $R$-configuration), which suggests that small ESP fields affect radiosensitizing activity.

The TX-2036 derivative has two characteristic structures, a 2-nitroimidazole site and a 4-cyclopentene-1,3-dione site.
We developed TX-1877 derivatives with a 2-nitroimidazole as the main framework and reported the correlation between structure and radiosensitizing activity $(9,10)$. For the TX2036 derivative, the 4-cyclopentene-1,3-dione structure may play a role in functional modification via small ESP fields. For this reason, we are investigating the radiosensitizing activity of a series of molecules with modifications in the 4-cyclopentene-1,3-dione structure. 


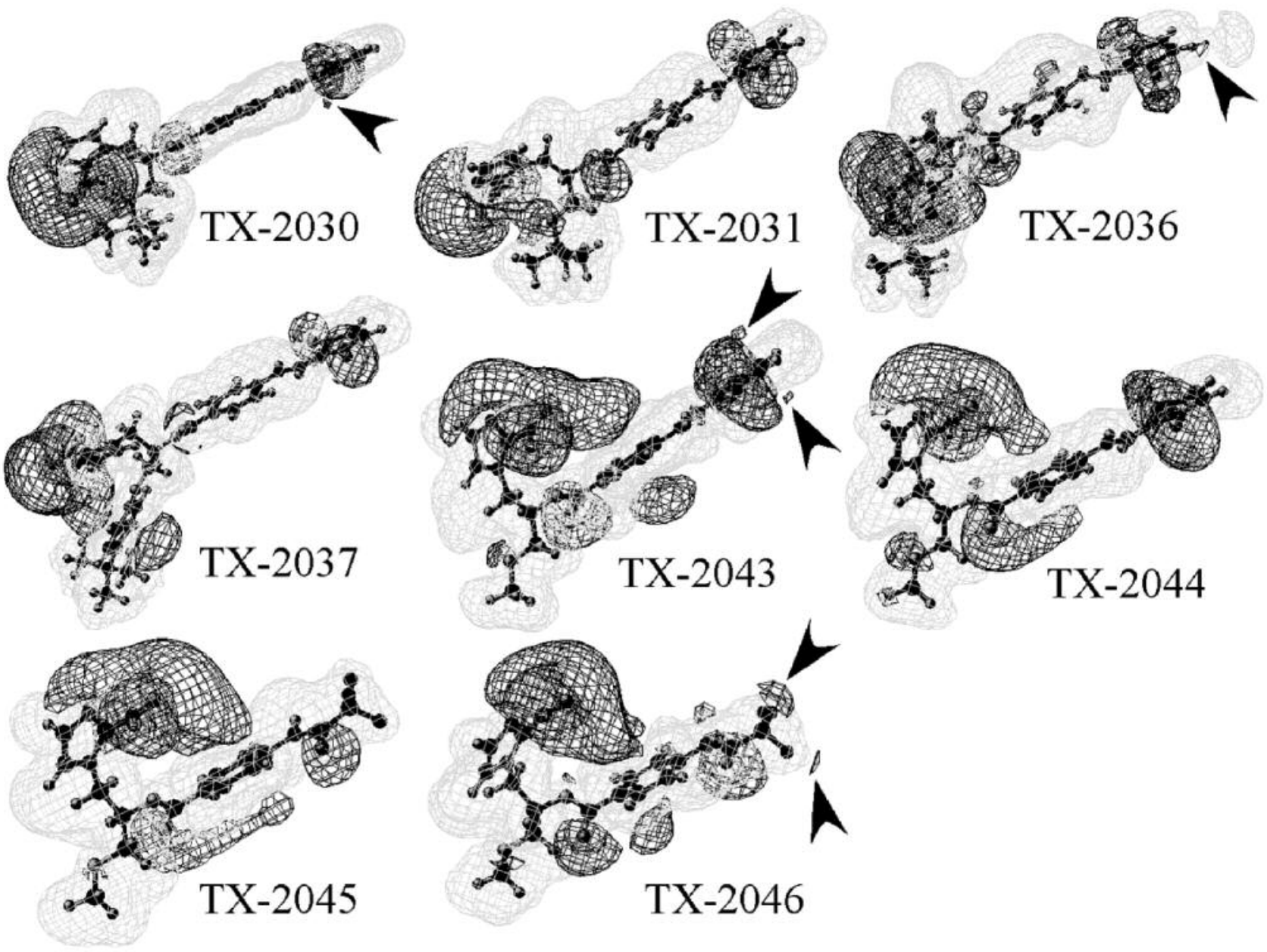

Figure 3. Electrostatic potential field of TX-2036 derivatives. Positive and negative fields are shown as gray and dark gray clouds, respectively. Small minus electrostatic potential fields are shown as arrowheads.

\section{Conflicts of Interest}

The Authors declare no conflicts of interest regarding this study.

\section{Acknowledgments}

This work was supported, in part, by a Grant-in-Aid for Scientific Research (18K06764) from the Ministry of Education, Culture, Sports, Science and Technology of the Japanese Government.

\section{Authors' Contributions}

Kazuto Ohkura, Atsushi Tabata and Yoshihiro Uto carried out the experiment. Kazuto Ohkura wrote the manuscript. Hitoshi Hori helped supervise the project. All Authors provided critical feedback and helped shape the research, analysis and manuscript.

\section{References}

1 Jin $\mathrm{CZ}$, Nagasawa $\mathrm{H}$, Shimamura $\mathrm{M}$, Uto $\mathrm{Y}$, Inayama $\mathrm{S}$, Takeuchi Y, Kirk KL and Hori H: Angiogenesis inhibitor TX1898: syntheses of the enantiomers of sterically diverse haloacetylcarbamoyl-2-nitroimidazole hypoxic cell radiosensitizers. Bioorg Med Chem 12: 4917-4927, 2004. PMID: 15336271. DOI: $10.1016 /$ j.bmc.2004.06.039

2 Uto Y, Nagasawa H, Jin CZ, Nakayama S, Tanaka A, Kiyoi S, Nakashima H, Shimamura M, Inayama S, Fujiwara T, Takeuchi Y, Uehara Y, Kirk KL, Nakata E and Hori H: Design of antiangiogenic hypoxic cell radiosensitizers: 2-nitroimidazoles containing a 2-aminomethylene-4-cyclopentene-1,3-dione moiety. Bioorg Med Chem 16: 6042-6053, 2008. PMID: 18474428. DOI: 10.1016/j.bmc.2008.04.041

3 Ohkura K, Sukeno A, Yamamoto K, Nagamune H, Maeda T and Kourai $\mathrm{H}$ : Analysis of structural features of bis-quaternary 
ammonium antimicrobial agents 4,4'-(alpha,omega-polymethylenedithio)bis(1-alkylpyridinium iodide)s using computational simulation. Bioorg Med Chem 11: 5035-5043, 2003. PMID: 14604666. DOI: 10.1016/j.bmc.2003.08.027

4 Ohkura K, Sukeno A, Nagamune H and Kourai H: Bridgelinked bis-quaternary ammonium anti-microbial agents: relationship between cytotoxicity and anti-bacterial activity of 5,5'-[2,2' -(tetramethylenedicarbonyldioxy)-diethyl] bis (3alkyl-4-methylthiazonium iodide)s. Bioorg Med Chem 13: 2579-2587, 2005. PMID: 15755659. DOI: 10.1016/j.bmc. 2005.01.030

5 Ohkura K and Hori H: Analysis of structure-permeability correlation of nitrophenol analogues in newborn rat abdominal skin using semiempirical molecular orbital calculation. Bioorg Med Chem 7: 309-314, 1999. PMID: 10218822. DOI: 10.1016/ S0968-0896(98)00200-4

6 Zhu JW, Nagasawa H, Nagura F, Mohamad SB, Uto Y, Ohkura $\mathrm{K}$ and Hori $\mathrm{H}$ : Elucidation of strict structural requirements of brefeldin a as an inducer of differentiation and apoptosis. Bioorg Med Chem 8: 455-463, 2000. PMID: 10722169. DOI: 10.1016/ S0968-0896(99)00297-7

7 Ohkura K, Hori H and Shinohara Y: Role of C-terminal region of yeast ADP/ATP carrier 2 protein: dynamics of flexible Cterminal arm. Anticancer Res 29: 4897-4900, 2009. PMID: 20032454 .
8 Shibamoto Y, Ono K, Takahashi M, Kano E, Hori H, Shibata T, Inayama $\mathrm{S}$ and $\mathrm{Abe} \mathrm{M}$ : An in vitro and in vivo screening system for new hypoxic cell radiosensitizers using EMT6 cells. Jpn J Cancer Res 77: 1027-1033, 1986. PMID: 3096918.

9 Ohkura K, Kawaguchi Y, Tatematsu Y, Tabata A, Uto Y and Hori $\mathrm{H}$ : Structure-associated functional control of TX-1877 series by glyco-conjugation. Anticancer Res 38: 4241-4245, 2018. PMID: 29970557. DOI: 10.21873/anticanres. 12720

10 Miyake K, Shimada M, Nishioka M, Sugimoto K, Batmunkh E, Uto $\mathrm{Y}$, Nagasawa $\mathrm{H}$ and Hori $\mathrm{H}$ : The novel hypoxic cell radiosensitizer, TX-1877 has antitumor activity through suppression of angiogenesis and inhibits liver metastasis on xenograft model of pancreatic cancer. Cancer Lett 272: 325-235, 2008. PMID: 18762366. DOI: 10.1016/j.canlet.2008.07.020

Received May 23, 2019

Revised June 18, 2019

Accepted June 19, 2019 